\title{
Effects of surface roughness on shear viscosity
}

\author{
Michail Papanikolaou ${ }^{1}$, Michael Frank ${ }^{2 *}$, Dimitris Drikakis ${ }^{2}$ \\ ${ }^{1}$ Cranfield University, Cranfield, MK43 OAL, UK \\ ${ }^{2}$ University of Strathclyde, Glasgow, G1 $1 X J, U K$
}

\begin{abstract}
This paper investigates the effect of surface roughness on the fluid viscosity using Molecular Dynamics simulations. The three-dimensional model consists of liquid argon flowing between two solid walls whose surface roughness was modelled using fractal theory. In tandem with previously published experimental work, our results show that, while the viscosity in smooth channels remains constant across the channel width, in the presence of surface roughness it increases close to the walls. The increase of the boundary viscosity is further accentuated by an increase in the depth of surface roughness. We attribute this behaviour to the increased momentum transfer at the boundary, a result of the irregular distribution of fluid particles near rough surfaces. Furthermore, although the viscosity in smooth channels has previously been shown to be independent of the strength of the solidliquid interaction, here we show that in the presence of surface roughness, the boundary viscosity increases with the solid's wettability. The paper concludes with an analytical description of the viscosity as a function of the distance from the channel walls, the walls' surface roughness, and the solid's wetting properties. The relation can potentially be used to adjust the fluid dynamics equations for a more accurate description of microfluidic systems.
\end{abstract}

\section{Introduction}

Owing to an increasing number of potential applications, the field of micro- and nano-fluidics is becoming an area of significant academic interest. A body of experimental and computational work has shown that under spatial restrictions of micro- and nano-meter characteristic lengths, the fluid properties and flow field differ significantly compared to their macroscopic counterparts. Therefore, models that bridge discrepancies between disparate scales are required for the design of optimal micro- and nano-fluidic devices.

So far, much of the attention has been turned on the boundary velocity in nanochannels. Investigations considering atomically smooth channel walls provided evidence indicating that the no-slip condition, frequently employed in Computational Fluid Dynamics (CFD) simulations, is often inaccurate $[1,2,3,4,5]$. Subsequent studies have shown that under more realistic conditions where surface roughness is considered, the velocity close to the channel walls diminishes $[6,7,8,9,10]$. The different behaviour between smooth and rough channel walls has been attributed to the increased momentum transfer at the boundary layer [11], a result of the chaotic distribution and motion of liquid atoms next to irregular geometries [10].

A related yet relatively unexplored area is the nature of the viscosity of confined liquids. Past simulations have shown that that the shear stress and viscosity are anisotropic and that the viscosity increases closer to the channel walls [12, 13]. Furthermore, experiments have shown that in micro-channels, the viscosity close to the channel walls increases with the depth of surface roughness, an observation which led to analytical models for the viscosity as a function of the depth of surface roughness and distance from the channel walls $[11,14]$. 
Molecular Dynamics (MD) studies further examined how the nature of the surface geometry affects viscosity. An MD investigation used sinusoidal-shaped walls to model surface roughness in order to study the Couette flow of hexadecane through a channel [15]. The conclusion was that the viscosity increases with increasing amplitude of the sine-wave, i.e. depth of roughness, while it decreases marginally with increasing wavelength. Subsequent MD studies considered the effect of rectangular protrusions and concluded that the length of the protrusions affects the viscosity [6].

Modeling surface roughness as a single periodic function is effective in selectively studying the relation of specific parameters on the viscosity. However, the morphology of realistic surfaces generally lacks such a high level of symmetry and is more appropriately described as stochastic irregularities that span a large range of scales. Therefore, studies have proposed the use of fractals as means for modeling realistic surface geometries [16, 17, 18]. In this investigation, we have used MD simulations to study the effect of realistic surface roughness on the viscosity of fluids close to the channel walls. Fractal theory was used for the design of the surface roughness, allowing us to capture the different scales and stochastic nature of solid surfaces.

Agreeing with the present literature, our results suggest that while the viscosity at the center of the channel remains unchanged, the boundary viscosity close to the solid surfaces increases with increasing roughness depth. Furthermore, we show that in the presence of surface roughness the boundary viscosity increases with the strength of the solid-liquid interactions, a dependence that is absent in smooth channels. This knowledge guided the derivation of an analytical relation that calculates the viscosity in nano-channels as a function of the distance from the channel wall, the average depth of roughness, and the strength of the solid-liquid interactions. This relation is essentially a mesoscale model of the near-wall region, which can be used to refine continuum simulation models, thus allowing a more accurate description of near-wall micro flows .

\section{Simulation Method}

Our model consists of liquid argon confined by two silver walls. The dimensions of the simulation box in the $x, z$ and $y$ directions are $L_{x}=L_{z}=7.4 \mathrm{~nm}$, and $L_{y}=14.4 \mathrm{~nm}$ respectively. Prior to the inclusion of any roughness, the silver walls consist of eleven ( $\left.\begin{array}{lll}1 & 1 & 1\end{array}\right)$ Face Centred Cubic (FCC) lattice planes placed parallel to the $x \mathrm{z}$ plane and perpendicular to the y direction. The lattice constant was set to $4.086 \AA$ corresponding to the atomic spacing of silver. The open-source molecular dynamics simulator LAMMPS [16] was employed to perform the MD simulations.

The surface roughness was then modeled using the multivariate Weierstrass-Mandelbrot (WM) function $[19,20]$ : 


$$
\begin{aligned}
y(x, z)=C \sum_{m=1}^{M} & \sum_{n=0}^{n_{\max }} \gamma^{\left(D_{s}-3\right)}\left\{\cos \Phi_{m, n}\right. \\
& \left.-\cos \left[\frac{2 \pi \gamma^{n} \sqrt{\left(x^{2}+z^{2}\right)}}{L_{M A X}} \cdot \cos \left(\tan ^{-1}\left(\frac{z}{x}\right)-\frac{\pi m}{M}\right)+\Phi_{m, n}\right]\right\}
\end{aligned}
$$

The function embeds $M$ surfaces, each of which is a superposition of $\mathrm{n}_{\max }$ different frequencies. Each frequency on each surface, indexed by the integers $n$ and $m$ respectively, is offset by a random phase $\Phi_{m, n}$. The value of $\gamma$ defines the frequency density and $L_{M A X}$ is the size of the sample; in our case $L_{M A X}=L_{x}=L_{z}$. The parameter $D_{s}$ is the fractal dimension, an indication of the fractal's ability to fill up space. For a three-dimensional fractal, $2<D_{s}<$ 3. Finally, $C$ is a scaling factor and determines the average amplitude of the waves. It is defined by the equation

$$
C=L_{M A X}\left(\frac{G}{L_{M A X}}\right)^{D_{s}-2}\left(\frac{\ln \gamma}{M}\right)^{1 / 2}
$$

where $G$ is called the roughness parameter, a parameter of interest to this investigation as it can be adjusted to obtain different depths of roughness.

In the theoretical limit, a perfect fractal corresponds to an infinite number of frequencies, i.e. $n_{\max } \rightarrow \infty$. However, a more practical value can be selected by using the relation

$$
n_{\max }=\left[\frac{\log \left(L_{\max } / L_{\min }\right)}{\log \gamma}\right]
$$

where $L_{\min }$ is the smallest wavelength used in the system. Equation 3 ensures that the used wavelengths span the entire range from $L_{\min }$ to $L_{\max }$. The frequency density can generally take any value greater than one. Here, we use $\gamma=1.5$, which does not result in repeated wavelengths (another commonly used value is $\gamma=5$ ). For the fractal dimension, we have used $D_{s}=2.5$, which corresponds to a highly irregular topography.

For the roughness parameter, we have chosen the values $G=0, G=0.75$ and $G=1.5$, with $G=0$ corresponding to an atomically smooth wall and $G=1.5$ corresponding to the geometry with greatest depth of roughness. Starting with the atomically perfect planes and a centre-plane, we calculate the W-M function. We then remove all solid atoms that are on the side of the liquid with respect to this function (Fig. 1). Using this procedure, we first create the lower wall. The upper wall is then a mirror image obtained through reflection about the $\mathrm{x}-\mathrm{z}$ plane of the simulation box. 


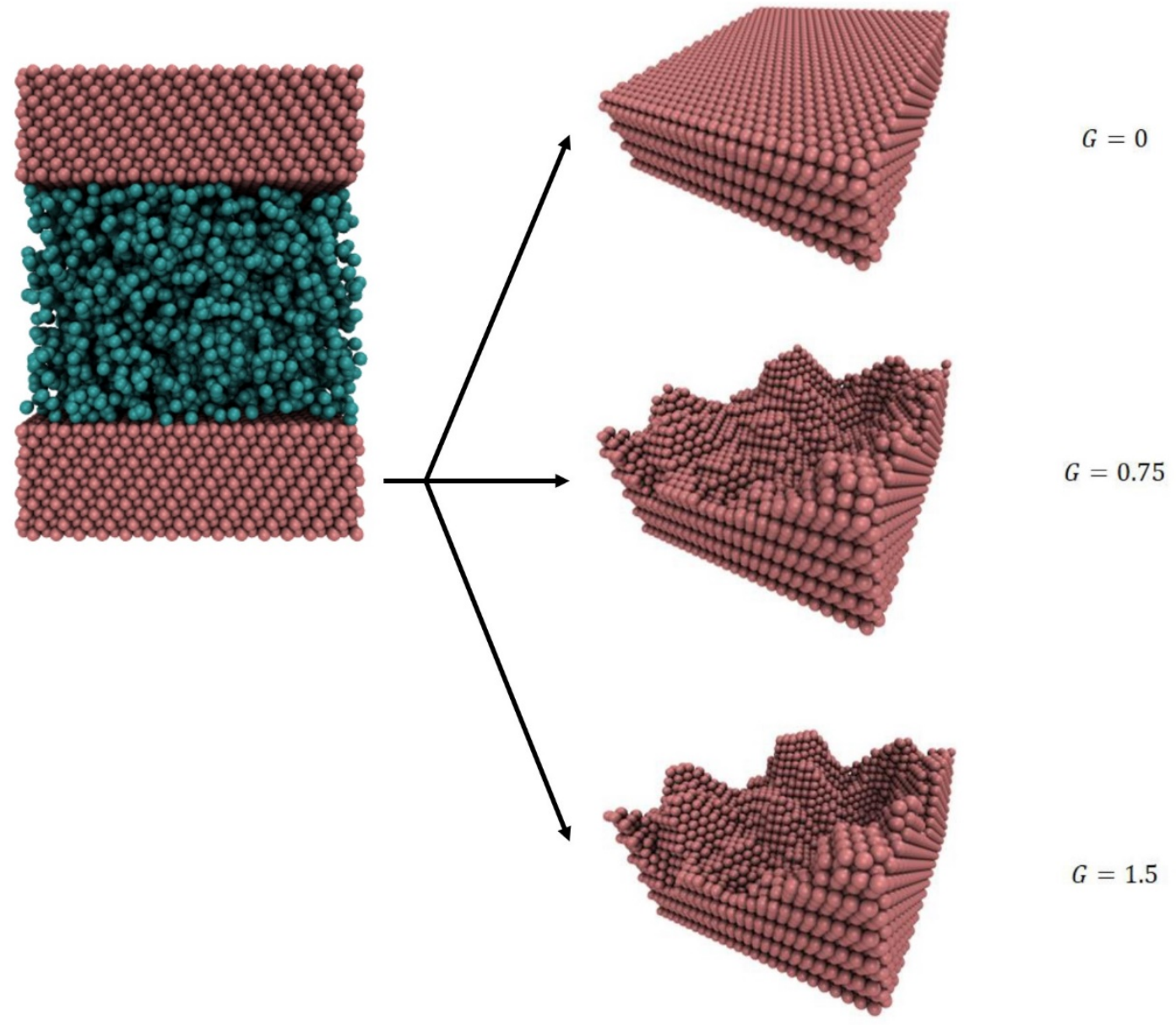

Fig. 1: MD model illustrating liquid argon (cyan) confined by two solid walls (pink). The surfaces to the right show walls of different values of the roughness parameter corresponding to a) $G=0, b$ ) $=0.75$ and c) $G=1.5$, respectively

The liquid atoms are then placed randomly between the walls; minimization of energy is performed in order to avoid any overlapping of the atoms. Since the volume of the channel differed significantly between cases of different roughness, we used dynamic Voronoi tessellation to estimate the available volume and in turn, insert a correct number of atoms to yield a constant density equal to $\rho=1.39 \mathrm{~g} / \mathrm{cm}^{3}$.

The wall-fluid and fluid-fluid interactions were modeled using the 12-6 Lennard-Jones (LJ) potential

$$
v_{i j}^{L J}=4 \varepsilon\left[\left(\frac{\sigma}{r_{i j}}\right)^{12}-\left(\frac{\sigma}{r_{i j}}\right)^{6}\right]
$$

where $\varepsilon$ is the depth of the potential well and quantifies the strength of the interaction; $\sigma$ is the van der Waals radius; and $r_{i j}$ is the distance between particles $i$ and $j$. The wall atoms were modeled using the EAM potential:

$$
v_{i}^{E A M}=\sum_{j=1}^{N_{i}} v_{i j}+f\left(\rho_{i}\right)
$$

where $\rho_{i}$ is the local electron density and $f$ an embedding function. 
The LJ parameters for the interactions between the various elements are given in Table I. The silver and argon masses were set equal to $m_{A g}=107.9 \mathrm{~g} / \mathrm{mol}$ and $m_{A r}=39.948 \mathrm{~g} / \mathrm{mol}$ respectively.

\begin{tabular}{|c|c|c|}
\hline Interaction & $\sigma(\AA)$ & $\varepsilon(\mathrm{eV})$ \\
\hline $\mathrm{Ag}-\mathrm{Ag}$ & 2.551 & 0.408 \\
\hline $\mathrm{Ar}-\mathrm{Ar}$ & 3.405 & 0.0104 \\
\hline $\mathrm{Ar}-\mathrm{Ag}$ & 2.978 & 0.010 \\
\hline
\end{tabular}

Table I. Molecular interaction parameters

The temperature of the system was controlled entirely through Langevin thermostats applied on each of the four solid planes furthest from the liquid on both walls, which were set to $115 \mathrm{~K}$. We did not tamper with the liquid atoms as this can result in unphysical behaviour [15].

The Verlet method was used to integrate Newton's laws of motion and the timestep used was equal to $5 \cdot 10^{-2}$ ps. Following an initial equilibration phase, the simulation was performed for $7 \times 10^{6}$ timesteps to calculate the liquid properties.

The Mean Square Displacement $(M S D)$ of the liquid atoms, used here for a qualitative understanding of the diffusive properties of the liquid, was calculated in the micro canonical (NVE) ensemble by

$$
\left\langle|\boldsymbol{r}(t)-\boldsymbol{r}(0)|^{2}\right\rangle=M S D(t)=\frac{1}{N} \sum_{i=1}^{N}\left(\left|\boldsymbol{r}_{i}(t)-\boldsymbol{r}_{i}(0)\right|^{2}\right)
$$

Where $N$ is the total number of liquid atoms in the system; $\boldsymbol{r}_{i}(0)$ is the position of the $i^{\text {th }}$ liquid atom at the beginning of the calculation (after the equilibration phase); and $\boldsymbol{r}_{i}(t)$ is the position of the atom at time $t$.

The spatial variation of the shear viscosity was measured by dividing the nano-channel into 100 bins across the width of the channel (y-direction), resulting in a bin-width of $1 \AA$. The pressure in each bin was estimated using equation 8. Due to the complex geometry of the walls, we used Voronoi tessellation to calculate the volume of each bin $\left(V_{b i n}\right)$. 


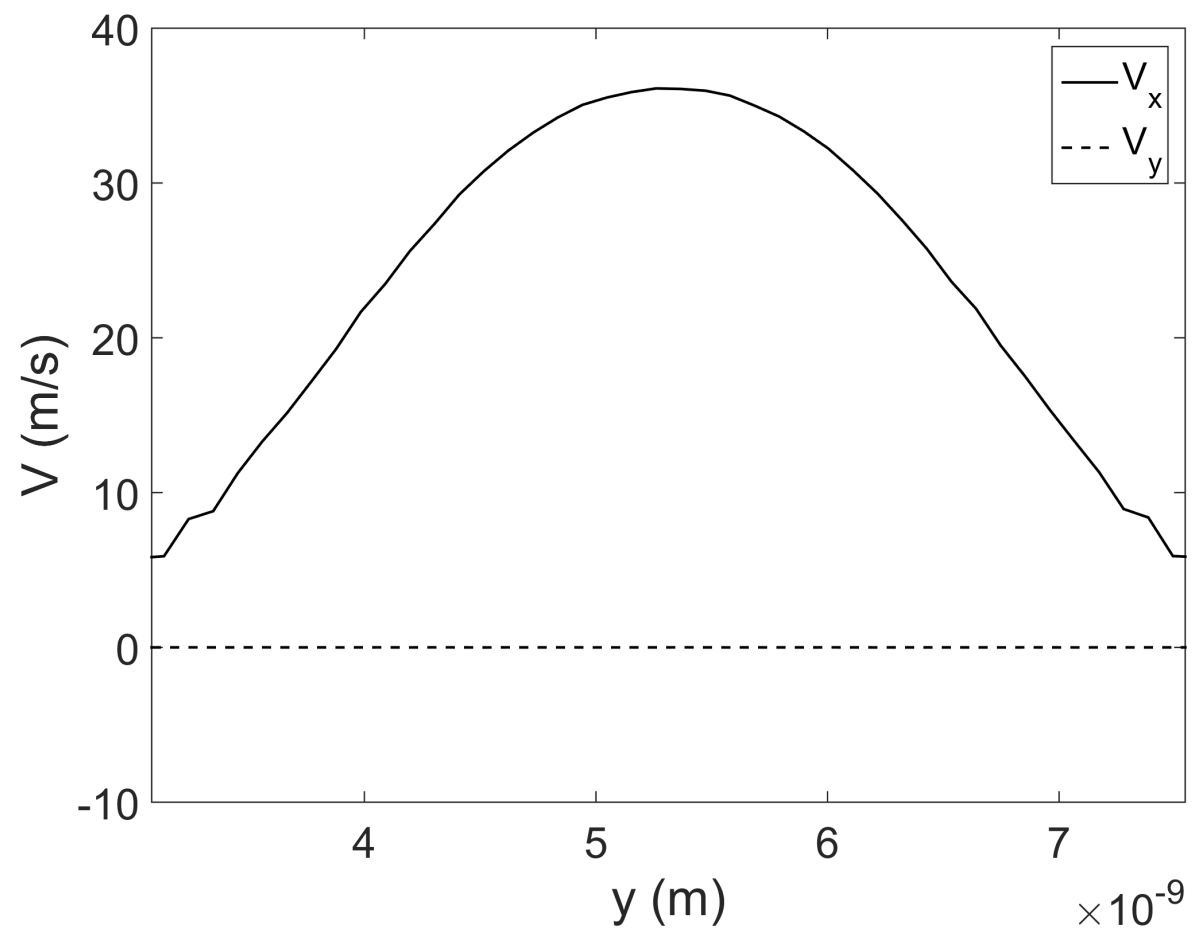

Fig. 2: Velocity profiles in the $x$ and $y$ channel directions.

For the calculation of the viscosity we used non-equilibrium molecular dynamics. After equilibration, we applied a force equal to $10^{-5} \mathrm{Kcal} /(\mathrm{mole} \cdot \AA)$ on each fluid particle to develop a Poiseuille flow in the $\mathrm{x}$-direction. The viscosity was then calculated by

$$
\eta=\frac{P_{x y}}{\dot{\gamma}}
$$

where $P_{x y}$ is the component of the symmetric pressure tensor acting in the $x$ direction on a plane normal to the $y$ direction, and $\dot{\gamma}$ is the shear rate. The shear rate is given by $\dot{\gamma}=\frac{\partial V_{x}}{\partial y}+$ $\frac{\partial V_{y}}{\partial x}$, where $V_{x}$ and $V_{y}$ are the velocity components in the $x$ and $y$ direction, respectively. The velocity profiles of $V_{x}$ and $V_{y}$ (Fig. 2) have been obtained by averaging the velocities across the bins in the $x$ - and z-directions; the $V_{y}$ component is of the order of $10^{-6}$, i.e. its contribution is negligible compared to $V_{x}$. Note that the values of $V_{y}$ are also of the order of $10^{-6}$ across each bin in the $x$ direction. Therefore, $\frac{\partial V_{y}}{\partial x}$ is also negligible.

The pressure tensor of Eq. (7) is obtained from the virial theorem, which calculates the pressure at the boundary of a volume, by averaging the thermal energy and interatomic forces acting on the liquid atoms contained within the volume. The component of the symmetric pressure tensor $P_{x y}$ is given by [21]

$$
P_{x y}=\frac{1}{V_{b i n}}\left[N k_{b} T+\frac{1}{2} \sum_{i=1}^{N} \sum_{j}^{N_{p}} r_{i j x} F_{i j y}\right]
$$

where $r_{i j x}$ is the interatomic distance between atoms $i$ and $j$, and $F_{i j y}$ is the component of the force in the $y$ direction between the two atoms. The summation over $i$ iterates over all the liquid atoms contained in the volume $V_{\text {bin }}$. The summation over $j$ iterates over all the 
neighbors of atom $i$, including atoms outside the volume, as well solid atoms. Therefore, the calculated stress is that of the liquid atoms in the volume due to their interaction with all other atoms within the cut-off distance.

From a statistical-mechanical point of view, the virial stress is strictly correct in homogeneous systems in equilibrium. In practice, however, it is effective in calculating the pressure even in highly inhomogeneous systems [21], such as in the vicinity of shockwaves [22]. Furthermore, any errors due to inhomogeneities tend to smooth out in fluid systems because of the constant fluctuation of the atoms [23]. We take advantage of these fluctuations by time-averaging over a large number of timesteps $\left(7 \times 10^{6} \tau\right)$. Finally, errors due to inhomogeneities can be alleviated by increasing the number of included atoms [24]. The bins used in the calculation of the viscosity contained more than a hundred atoms, whereas bins containing a smaller number of atoms were neglected to avoid spurious oscillations.

\section{Results and Discussion}

The introduction and increasing depth of surface roughness results in significant changes in the fluid structure close to the channel walls. Under surface roughness, the localised, equally spaced, parallel liquid layers found next to smooth solid surfaces (Fig. 3(a)) transform into arbitrary shaped structures spread over a larger percentage of the channel width (Fig. 3(b)). This breakup of layers is a result of the anisotropic potential generated by the irregular geometry of the walls. We now show that this mixing of layers next to rough walls increases the viscosity closer to the solid surfaces.

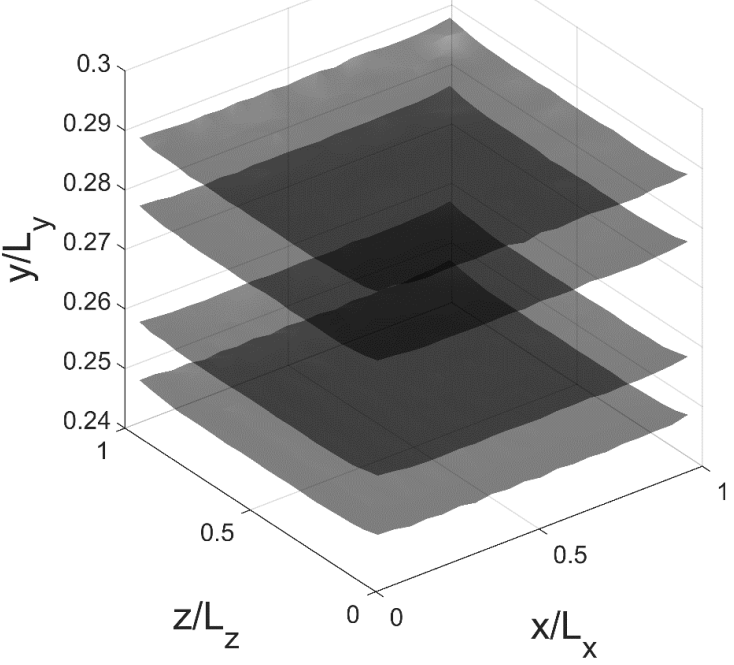

(a) $\mathrm{G}=0$

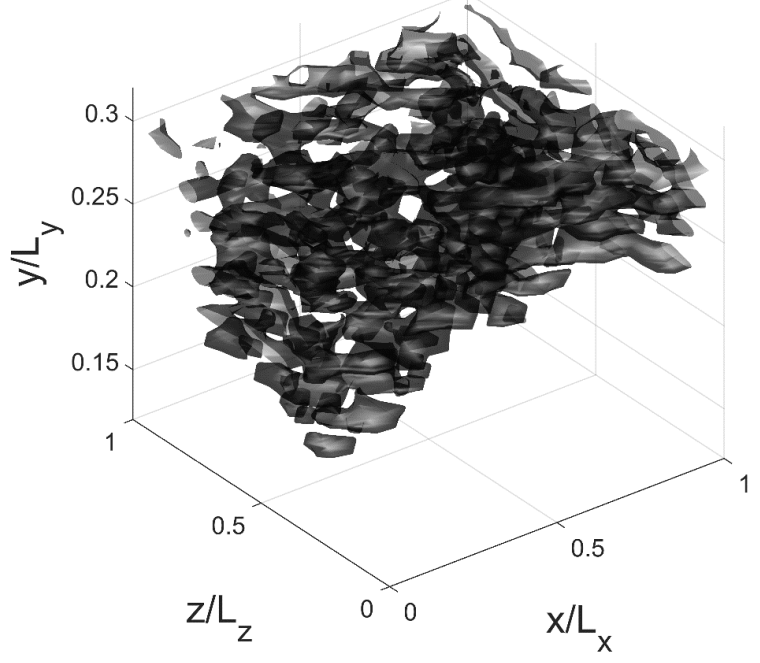

(b) $\mathrm{G}=1.75$

Fig. 3: Density isosurfaces for $\rho=1.525 \mathrm{~g} / \mathrm{cm}^{3}$ for (a) smooth and (b) rough channel walls

The MSD - and therefore diffusion coefficient - qualitatively demonstrates this increase in viscosity. The rate of change of the MSD in the $x$ direction (Fig. 4a) decreases with increasing depth of surface roughness, an indication of hindered atomic mobility (the zdirection exhibits identical behaviour). The viscosity, inversely proportional to the diffusion coefficient, must therefore increase with increasing depth of roughness. In accordance with the literature [12], the MSD in the y direction (Fig. 4b) quickly reaches a plateau due to the spatial restriction imposed by the channel. Therefore, it does not provide sufficient insight into the diffusion coefficient in the perpendicular direction. 


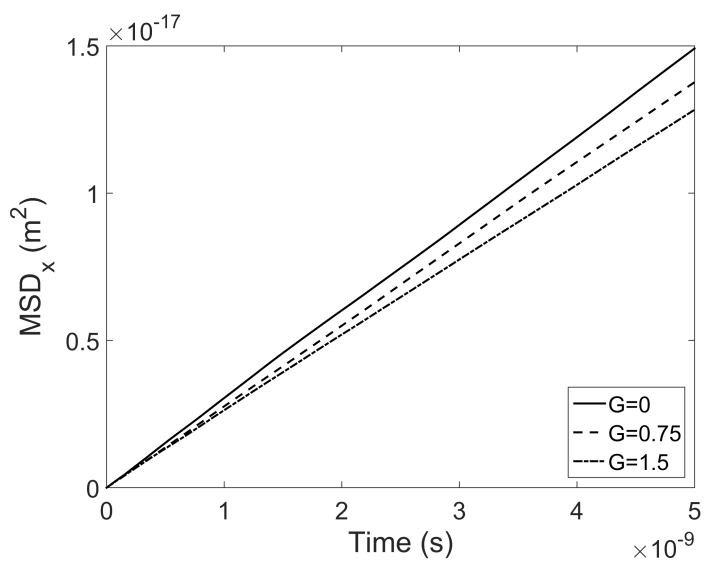

(a) $\mathrm{x}$-direction

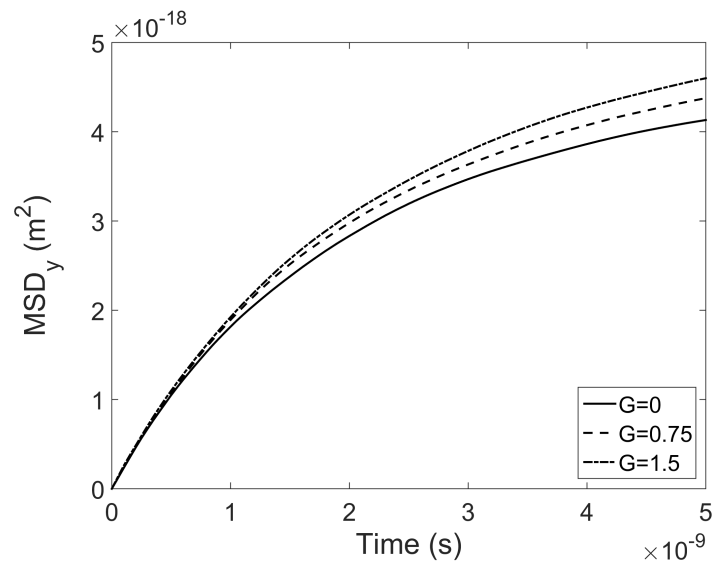

(b) y-direction

Fig. 4: Mean square displacement in the a) $x$, b) $y$ directions. The MSD in the $z$ direction is identical to that in the $x$ direction and was omitted.

With the introduction of surface roughness, we find that the strain rate, required for the calculation of the viscosity, exhibits a non-linear behaviour close to the channel walls and decreases with increasing depth of roughness (Fig. 5a). The shear stress also decreases between the three cases, albeit significantly less than the decrease of the strain rate (Fig. $5 \mathrm{~b}$ ). We attribute this slight decrease to the breakup of the structured layers close to the walls: the close packing of the liquid atoms in the structured layers results in stronger forces between them. As roughness breaks these layers, the interactions weaken and start to resemble those of free liquid atoms.

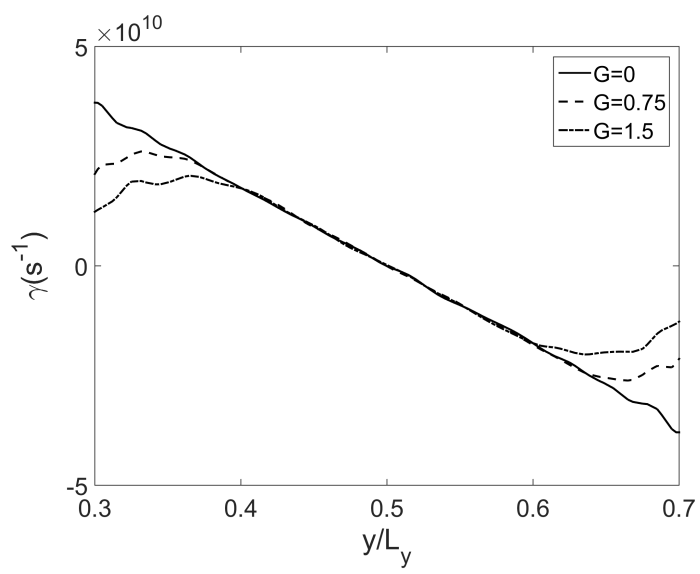

(a) Strain rate

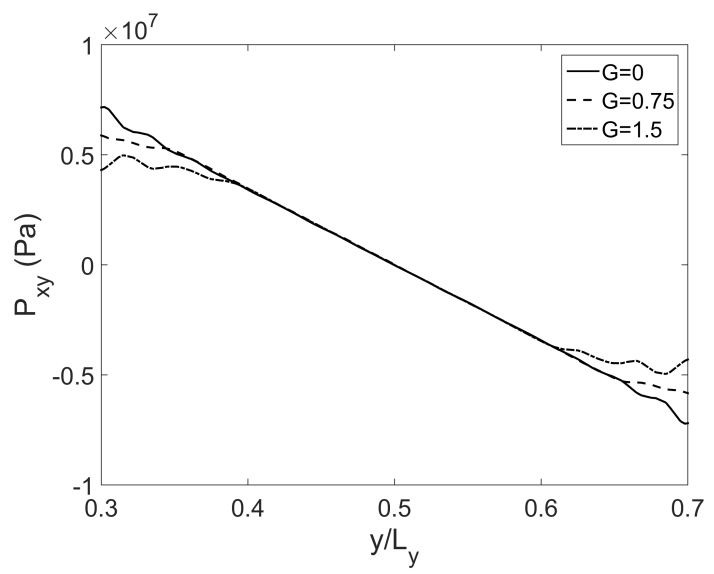

(b) Shear stress

Fig. 5 (a) Strain rate and (b) shear stress of the liquid across the channel width for different values of the average depth of roughness

In turn, we see that the viscosity close to the channel walls increases with increasing depth of roughness, an observation consistent with previous experimental results [11]. Furthermore, the viscosity decreases exponentially with the distance from the channel walls, and reaches the value of argon's bulk viscosity at the center of the channel.

We attribute the increasing boundary viscosity with increasing roughness to the immobile fluid atoms next to rough surfaces: In smooth channels the structured boundary layers occupy a separate region along the channel width, compared to that occupied by the free fluid particles. This allows the flowing atoms to "glide" over the boundary layer with minimal interaction. On the contrary, in rough channels, the boundary layers intrude the flow region. 
Therefore, flowing atoms collide with these liquid protrusions. Such collisions restrict fluid flow and in turn give rise to greater viscosity.

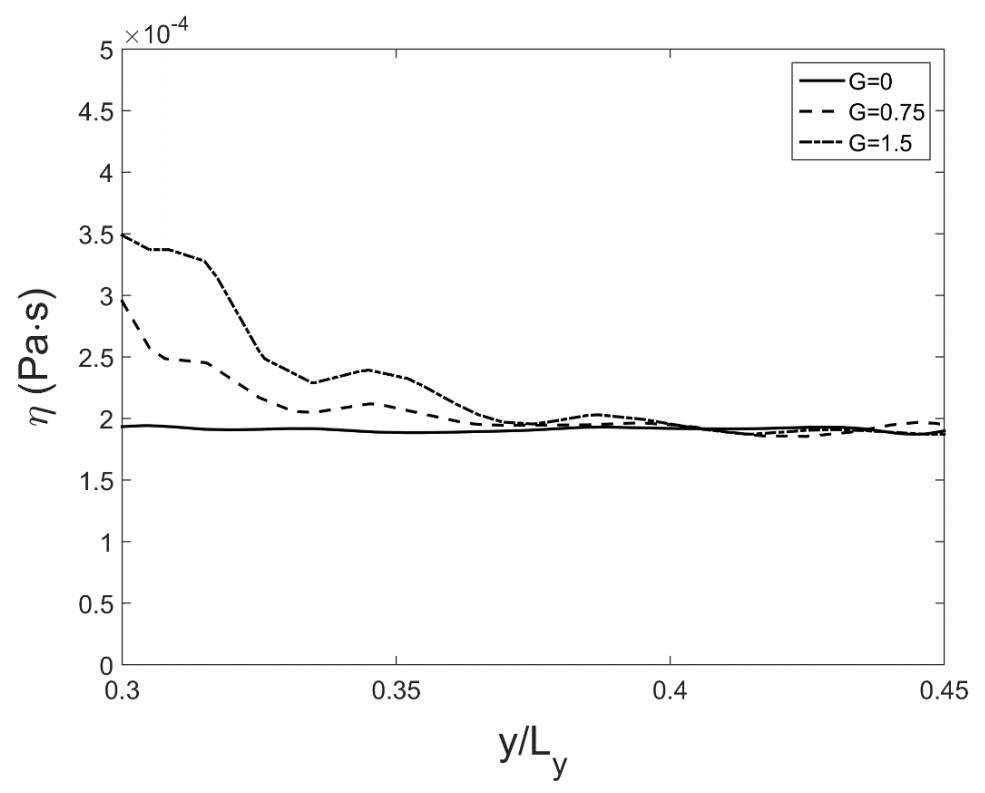

Fig. 6: Viscosity profiles for different values of the roughness parameter $G$

Finally, while the strength of the solid liquid interaction, $\varepsilon_{w f}$, does not affect the viscosity in smooth channels [25], we have found that it becomes increasingly important as the depth of roughness increases. This is because, as roughness is introduced, collisions with the immobile boundary structures are affected significantly by the binding energy of the boundary layer which increases with increasing $\varepsilon_{w f}$.

We therefore believe that current viscosity models, which do not take the solid-liquid interactions into account, are incomplete. Indeed, although previously derived relations qualitatively captured the exponential decay of the viscosity, they failed to accurately fit our data [11]. By adjusting this pre-existing model to account for the strength of the solid-liquid interactions, we concluded with the relation:

$$
\mu(y)=\mu_{b u l k}+\frac{A}{L_{y}{ }^{4}} G\left(\varepsilon_{w f}+2 \times 10^{-4}\right)\left|\left(\frac{L_{y}}{2}-y\right)\right|^{3}\left(1-e^{\left|\frac{L_{y}}{2}-y\right|}\right)^{2}
$$

where $\mu_{\text {bulk }}$ is the bulk shear viscosity of argon and $A=30 \frac{\mathrm{Pa} \cdot \mathrm{s}}{\mathrm{eV} \cdot \AA}$ is a constant. From the relation, the significance of the strength of the solid-liquid interaction decreases with decreasing roughness depth and completely diminishes in smooth channel walls. The expression captures our results very well, for cases of different roughness and wetting properties $\varepsilon_{w f}$.

To give credence to our viscosity model, we considered a Couette flow, initiated by translating the top wall at a velocity $300 \mathrm{~m} / \mathrm{s}$. The surface of the moving wall was smooth, as the motion of a fractal surface would result in viscous heating. The (lower) stationary wall was identical to the previous cases with $G=1.5$. The calculated viscosity profile is very similar to the corresponding Poiseuille-flow case and is well captured by Eq. (9) (Fig. 7d). 
Eq. 9 provides a mesoscale model of the viscosity in the near wall region, which can be used in conjunction with continuum CFD models to provide a more accurate description of micro flows in the vicinity of walls of micro and nano channels [26, 27, 28].

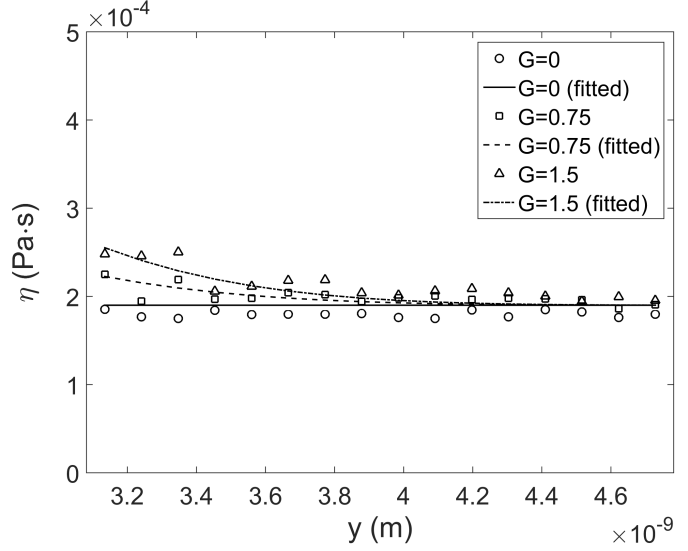

(a) $\varepsilon_{\mathrm{wf}}=0.002 \mathrm{eV}$

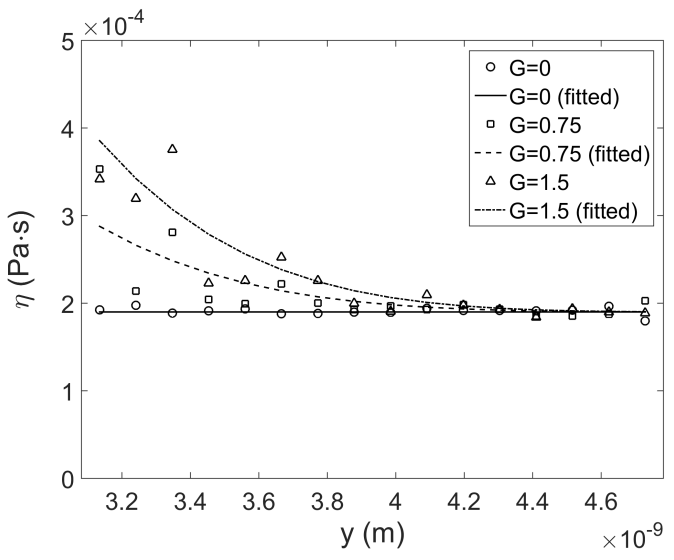

(c) $\varepsilon_{\mathrm{wf}}=0.01 \mathrm{eV}$

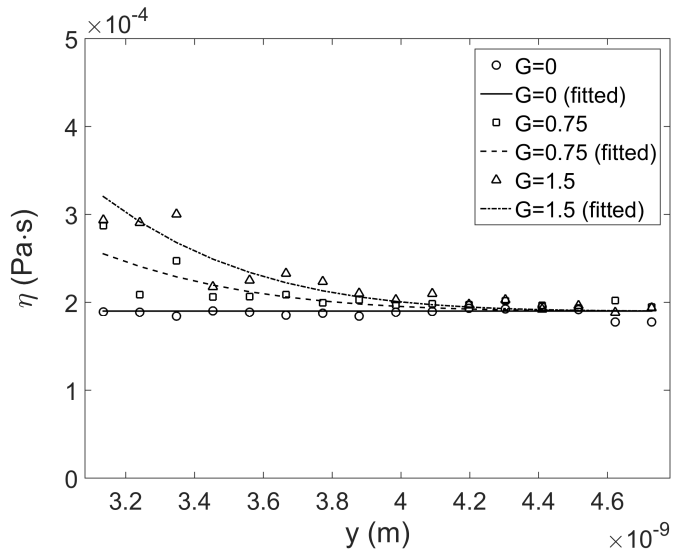

(b) $\varepsilon_{\mathrm{wf}}=0.006 \mathrm{eV}$

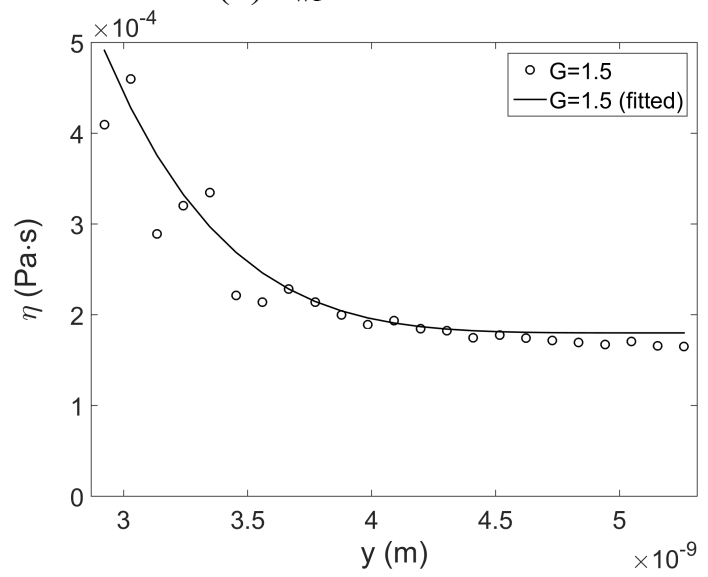

(d) $\varepsilon_{\mathrm{wf}}=0.01 \mathrm{eV}$ (Couette Flow)

Fig. 7: Viscosity profiles for different values of the roughness parameter $G$ for a) $\varepsilon_{w f}=0.002 \mathrm{eV}, \quad b$ ) $\varepsilon_{w f}=0.002 \mathrm{eV}, \mathrm{c}$ ) $\varepsilon_{w f}=0.010 \mathrm{eV}$, and $\varepsilon_{w f}=0.010 \mathrm{eV}$ (Couette Flow)

\section{Conclusions}

We have investigated the effect of realistic surface roughness on the boundary viscosity. In agreement with experimental results, we have found that the viscosity close to the channel walls increases with increasing depth of roughness. Moving away from the channel walls, the effect of roughness diminishes and becomes unimportant at the centre of the channel, where the viscosity of bulk argon is realised. Furthermore, although previous investigations have found that, in smooth channels, the viscosity is unaffected by the solid's wetting properties, here we show that under surface roughness the strength of the solid-liquid interactions affects the boundary viscosity and becomes increasingly important as the depth of roughness increases. The information obtained from the simulations was moulded into an analytical relation expressing the viscosity in micro- and nano-channels as a function of the distance from the wall, the depth of roughness, and the strength of the solid-liquid interaction. The expression can potentially be used to tailor the momentum and energy equations of fluid dynamics for micro- and nanofluidics. 


\section{Bibliography}

[1] N. Asproulis and D. Drikakis, Phys. Rev. E, 81, 061503 (2010).

[2] R. S. Voronov, D. V. Papavassiliou and L. L. Lee, Chem. Phys. Let, 441,273, 2007.

[3] N. Asproulis and D. Drikakis, Phys. Rev. E, 84, 031504 (2011).

[4] C. Soong, T. Yen and P. Tzeng, Phys. Rev. E, 76, 036303 (2007).

[5] S. K. Prabha and S. P. Sathian, Phys. Rev. E, 85, 041201 (2012).

[6] F. Sofos, T. E. Karakasidis and A. Liakopoulos, Int. J. Heat Mass Transfer, 53, 3839 (2010).

[7] N. Asproulis and D. Drikakis, J. Comput. Theor. Nanosci., 7, 1825 (2010).

[8] C. Zhang and Y. Chen, Chem. Eng. Process.: Process Intensification, 85, 203(2014).

[9] Y. Chen, C. Zhang, M. Shi and G. P. Peterson, App. Phys. Lett., 100, 074102(2012).

[10] M. Papanikolaou, M. Frank and D. Drikakis, Phys. Fluids, 28, 082001(2016).

[11] C. Merkle, T. Kubota and D. Ko, "An analytical study of the effects of surface roughness on boundary-layer transition," Defense Technical Information Center.

[12] F. Sofos, T. Karakasidis and A. Liakopoulos, Intern. J. Heat Mass Transfer, 52, 735(2009).

[13] R. Hartkamp, A. Ghosh, T. Weinhart and S. Luding, J. Chem. Phys., 137, 044711(2012).

[14] G. M. Mala and D. Li, Int. J. Heat Fluid Fl, 20, 142(1999).

[15] A. Jabbarzadeh, J. Atkinson and R. Tanner, Phys. Rev. E, 6, 690 (2000).

[16] A. Majumdar and B. Bhushan, J. Tribol., 112, 205, (1990).

[17] S. Hyun, L. Pei, J.-F. Molinari and M. O. Robbins, Phys. Rev. E, 70, 026117 (2004).

[18] Y. Chen, C. Zhang, M. Shi and G. Peterson, Phys. Rev. E, 80, 026301 (2009).

[19] M. Ausloos and D. Berman, P. Roy. Soc. A, 400, 331(1985).

[20] B. Chatterjee and P. Sahoo, Procedia Eng., 90, 116(2012).

[21] A. P. Thompson, S. J. Plimpton and W. Mattson, J. Chem. Phys., 131154107(2009).

[22] J. Budzien, A. P. Thompson and S. V. Zybin, J. Phys. Chem. B, 113, 13142(2009).

[23] K. S. Cheung and S. Yip, J. Appl. Phys., 70, 5688(1991). 
[24] D. Tsai, J. Chem. Phys., 70, 1375(1979).

[25] P. A. Thompson and S. M. Troian, Nature, 389, 360(1997).

[26] A. Niavarani and N. V. Priezjev, Phys. Rev. E, 81, 011606(2010).

[27] A. Giannakopoulos, F. Sofos, T. Karakasidis and A. Liakopoulos, Int. J. Heat Mass Transfer, 55, 5087(2012).

[28] A. Liakopoulos, F. Sofos and T. E. Karakasidis, Microfluid Nanofluidics, 20, 24, 2016. 